\title{
Etude du salage et de l'affinage du fromage en saumure \\ III. - ASPECT BACTERIOLOGIQUE
}

par

\author{
A. MANSOUR et C. ALAIS
}

Ecole de Laiterie (ENSAIA) et Université de Nancy I - F 54 - Nancy

\section{I. - INTRODUCTION}

Dans les deux précédentes communications (Mansour et Alais, 1972, a, b) nous avons étudié les aspects biochimiques et physicochimiques du salage et de l'affinage en saumure et, en particulier, le problème des échanges de sels, d'acide lactique et de constituants protidiques entre le fromage et la saumure, dans différentes conditions de conservation.

Les études microbiologiques de ce type de fromage sont rares; elles ne concernent pas un examen systématique de la microflore mais sont plutôt en rapport avec l'altération du fromage. Abo-El Naga (1971) a ainsi attribué à Klebsiella aerogenes le gonflement du fromage " Domiati », au cours de l'affinage en saumure. Kiss et al. (1966) et Ghoniem (1968) ont montré que des levures peuvent être à l'origine d'accidents du même genre. Il faut d'ailleurs remarquer, en considérant les fromages des pays occidentaux, que, bien que beaucoup plus nombreux, les travaux de bactériologie fromagère concernent plus les causes d'anomalies ou d'altération que l'étude approfondie de la microflore normale du fromage. A ce sujet, citons les récents travaux de Devoyod et al. $(1968,1970)$ dont deux publications, relatives au fromage de Roquefort, nous ont particulièrement intéressés.

En tenant compte du rôle important que peut jouer la flore microbienne sur la qualité du fromage et sur celle de la saumure, nous nous sommes proposés de suivre l'évolution des germes totaux, de la flore lactique, des levures et des bactéries coliformes. Le nombre élevé de levures trouvées dans la saumure nous a conduits à faire une identification des espèces halophiles. 


\section{II. - MATERIEL ET METHODES}

Nous avons analysé le fromage conservé à $10^{\circ} \mathrm{C}$ et la saumure de conservation à 18 p. $100 \mathrm{de} \mathrm{NaCl}$. Nous avons également analysé le fromage conservé à $10^{\circ} \mathrm{C}$ sous film plastique (procédé Cryovac). Les techniques de préparation ont été précédemment décrites (Mansour et Alais, 1972, a ; Mansour, 1972).

Pour le fromage nous avons utilisé une suspension de fromage à 10 p. 100 dans la solution de Ringer ; elle est obtenue par l'OmniMixer Sorvall, dont les pots sont préalablement stérilisés. Pour la saumure, nous avons effectué l'analyse directement, sans préparation particulière.

Les dénombrements ont été faits par la technique des dilutions en utilisant les milieux suivants :

- Tryptone-agar, selon la norme F.I.L. pour la flore totale (voir réf. Serres, 1970).

- Milieu de Elliker et al., (1956) pour les streptocoques. bacilles.

- Milieu de de Man, Rogosa et Sharpe (1960) pour les lacto-

- Milieu O.G.A. à la terramycine pour les levures et moisissures (voir réf. Serres, 1970).

- Milieu lactosé aux sels biliaires et au vert brillant pour les coliformes.

L'identification des levures a été faite selon la méthodologie proposée par Lodder et Kreeger van Rij (1952). La composition des milieux utilisés et les techniques de manipulation ont déjà été décrites en détail (Mansour, 1972).

\section{III. - RESULTATS}

\subsection{Flore microbienne}

Le premier salage du fromage découpé provoque une réduction sensible du nombre des bactéries et surtout de la microflore dite «totale » (environ 3,5 fois), alors que les streptocoques sont réduits de 1,6 fois, comme le montre le tableau 1 .

Le fromage est fabriqué à partir de lait pasteurisé auquel on ajoute une culture de streptocoques lactiques (0,5 p. 100) ; c'est ce qui explique le nombre élevé de ces espèces, obtenu par culture sur le milieu riche de Elliker et al. (1956) et le fait qu'après présalage on dénombre plus de germes sur ce milieu que sur le milieu tryptoneagar. 

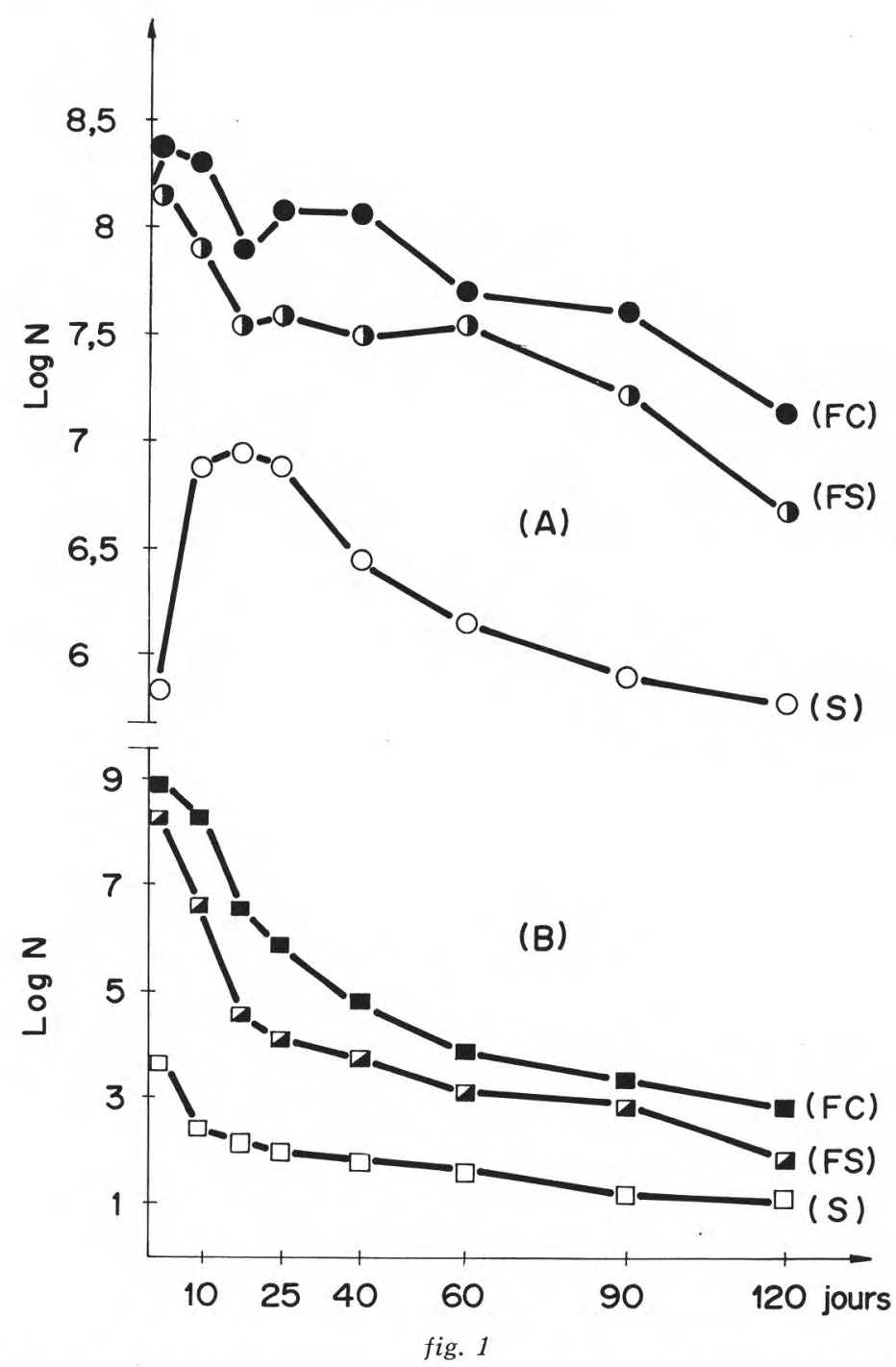

Evolution de la flore "totale » $(A)$ et de la flore lactique (B) dans le fromage conservé en saumure (FS), dans le fromage conservé sous film Cryovac (FC) et dans la saumure (S). (Log $N$ : logarithme du nombre de germe par $\mathrm{g}$ ou par $\mathrm{ml}$ ).

L'évolution du nombre de germes est représentée dans la figure 1 pour les germes «totaux » (A) et pour les streptocoques lactiques (B).

Dans le fromage conservé en saumure, on observe une diminution des deux microflores (courbes FS, figure 1) ; le nombre des strepto- 
TABLEAU 1. - Influence du présalage sur la microflore du caillé (germes/g)

\begin{tabular}{|c|c|c|c|c|}
\hline & $\begin{array}{c}\text { Germes } \\
\text { «totaux» }(*)\end{array}$ & $\begin{array}{l}\text { Streptocoques } \\
\text { lactiques (**) }\end{array}$ & Levures & Coliformes \\
\hline Caillé frais & $5,1 \times 10^{8}$ & $4 \times 10^{8}$ & 40 & 0 \\
\hline $\begin{array}{l}\text { Caillé salé } \\
\text { (6 h saumure } 12 \text { p. } 100)\end{array}$ & $1,5 \times 10^{5}$ & $2,5 \times 10^{s}$ & 30 & 0 \\
\hline
\end{tabular}

(*) Germes se développant sur le milieu tryptone-agar.

(**) Germes se développant sur le milieu de Elliker et al. (1956).

coques subit une réduction considérable et rapide ; après $25 \mathrm{j}$ il n'en reste que quelques milliers et après $120 \mathrm{j}$, la flore lactique a presque disparu. La réduction de la flore " totale " est plus progressive et moins poussée ; elle se stabilise vers 35 millions entre le $18^{\mathrm{e}}$ et le $60^{\mathrm{e}} \mathrm{j}$ puis elle décroît à nouveau jusque vers 6 millions après $120 \mathrm{j}$.

Dans le fromage conservé sous film plastique, il y a toujours plus de bactéries que dans le fromage en saumure (courbes FC fig. 1). L'évolution de la flore lactique est approximativement parallèle ; celle de la flore totale est quelque peu différente ; dans les premiers jours on observe une augmentation sensible, de 150 à 240 millions, suivie d'une décroissance irrégulière, avec une remontée entre le $18^{\mathrm{e}}$ et le $25^{\mathrm{e}} \mathrm{j}$. A la fin de la conservation il y a 2,5 fois plus de bactéries dans ce fromage (15 millions) que dans le précédent (6 millions).

Dans la saumure (courbes S, fig. 1) la flore totale s'accroît rapidement ; elle passe de 800000 à 10 millions environ du $1^{\text {er }}$ au $15^{\mathrm{e}} \mathrm{j}$; puis elle décroît régulièrement et revient au niveau du $1^{\mathrm{er}} \mathrm{j}$ à la fin de la conservation. Les streptocoques lactiques sont moins nombreux et ils disparaissent presque complètement dans ce milieu fortement salé.

Nous avons recherché les lactobacilles sur le milieu M.R.S. mais nous n'avons pas pu mettre en évidence leur présence ni dans les fromages, ni dans la saumure. Il est à noter que les ferments ajoutés au lait ne contenaient pas de lactobacilles.

Les bactéries coliformes paraissent être absentes des fromages et de la saumure ; les essais en milieu lactosé avec sels biliaires et vert brillant ont tous été négatifs. 


\subsection{Evolution des levures et des moisissures}

Au début de la conservation, on trouve très peu de levures (tab. 1) ; ensuite leur nombre augmente très rapidement pendant les $10 \mathrm{j}$ qui suivent la fabrication; on trouve alors dans le fromage sous film Cryovac environ 30 millions de levures. Dans le cas du fromage en saumure, cette augmentation continue jusqu'au $18^{\mathrm{e}} \mathrm{j}$, où l'on arrive à en trouver un nombre 2,3 fois plus élevé que le précédent (fig. 2). Dans la saumure elle-même, le nombre de levures devient très

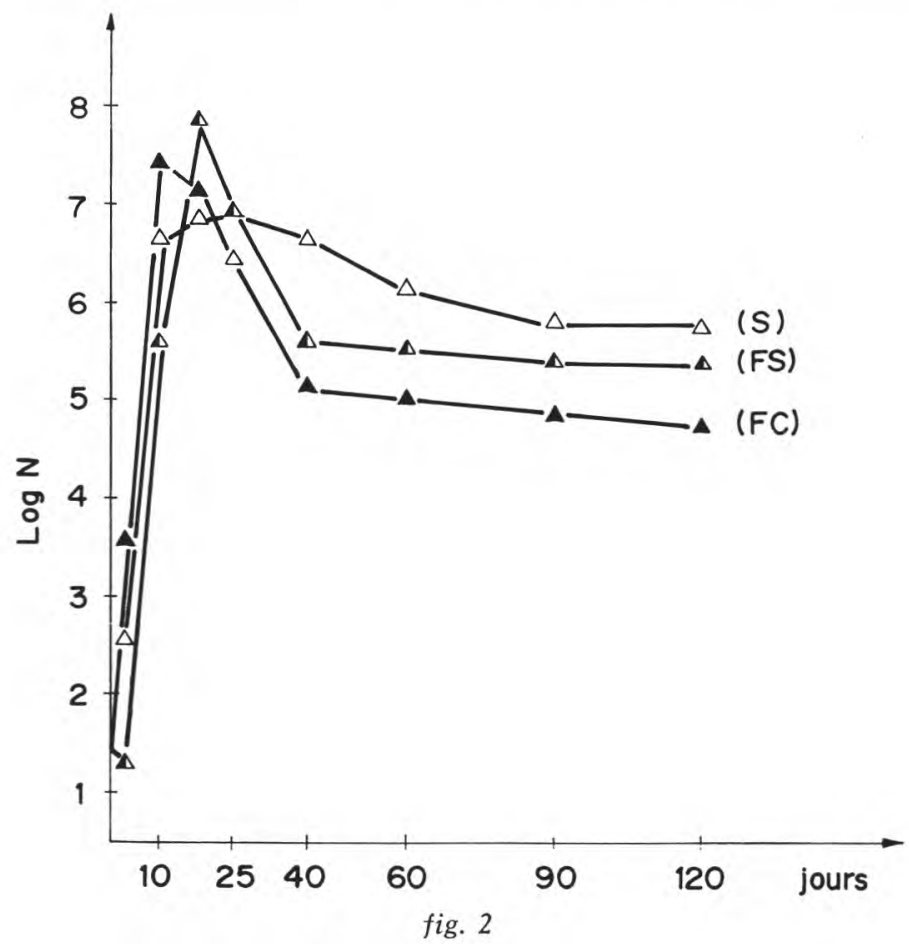

Evolution des levures (voir légende fig. 1)

élevé, il est maximal au $25^{\mathrm{e}} \mathrm{j}$. Après cette période de croissance, on constate que le nombre des levures commence à diminuer dans tous les cas, mais la décroissance est plus rapide dans le fromage sous Cryovac que dans le fromage salé, ou dans la saumure. A partir du $60^{\mathrm{e}}$ jour, le nombre de levures varie peu ; jusqu'à la fin de la conservation, on en trouve un nombre supérieur dans la saumure et dans le fromage salé par rapport au fromage sous Cryovac ; respectivement : 650000,260000 et 60000 levures.

Nous avons également compté les moisissures, en utilisant le même milieu que celui utilisé pour les levures ; le nombre trouvé au 
cours de la conservation était toujours inférieur à 100 pour $1 \mathrm{~g}$ de fromage ou $1 \mathrm{ml}$ de saumure. Il est certain que les conditions de conservation ne sont pas favorables à leur croissance. Par contre nous avons remarqué que les moisissures s'étaient développées, à la fin de la conservation, sur l'emballage Cryovac, du côté de la fermeture.

\subsection{Identification des levures survivant dans la saumure}

Neuf souches de levures ont été isolées à partir de la saumure, ayant 4 mois d'utilisation. Huit souches de ces levures possèdent la propriété de former des ascopores ; les cellules sont petites et ont une forme ronde cu légèrement ovale, comme le montre la figure 3.

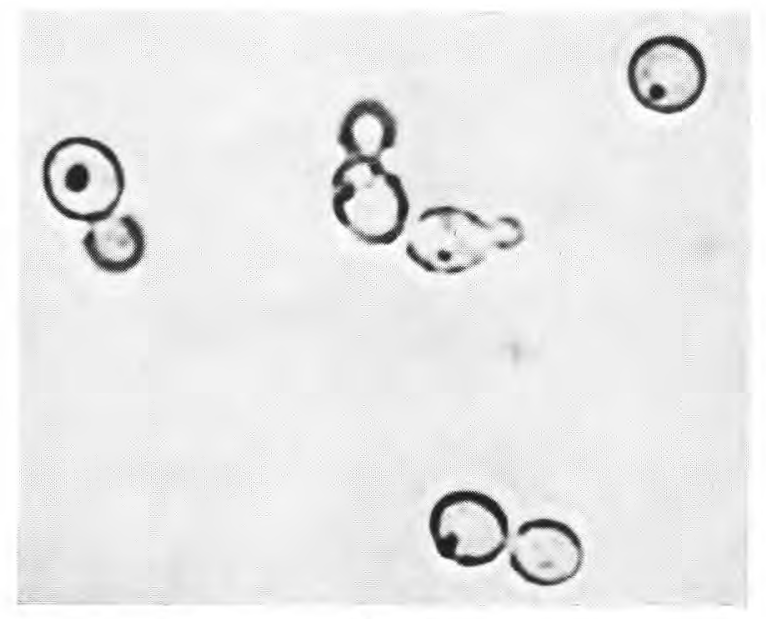

fig. 3

Debaryomyces hansenii, dans l'extrait de malt

$(3 \mathrm{j}$, grossissement $\times 400)$

Dans le tableau 2, nous avons regroupé les principaux caractères de ces levures. En suivant la classification proposée par Lodder et Kreeger Van Rij (1952), nous pouvons considérer ces souches comme appartenant au genre Debaryomyces.

Elles fermentent très faiblement le glucose, le galactose et le saccharose ; elles assimilent le glucose, le galactose, le saccharose, le maltose et le lactose ; mais elle n'assimilent pas les nitrates et elles forment rapidement des pellicules sur le milieu liquide. D'après ces propriétés morphologiques et physiologiques, il est probable qu'il s'agisse de l'espèce $D$. hansenii, appelée aussi D. subglobosus (Zach).

L'étude que nous avons faite sur la neuvième souche ne nous permet pas de la classer ; les résultats obtenus sont trop fragmentaires. 
TABLEAU 2. - Principaux caractères des levures isolées de la saumure

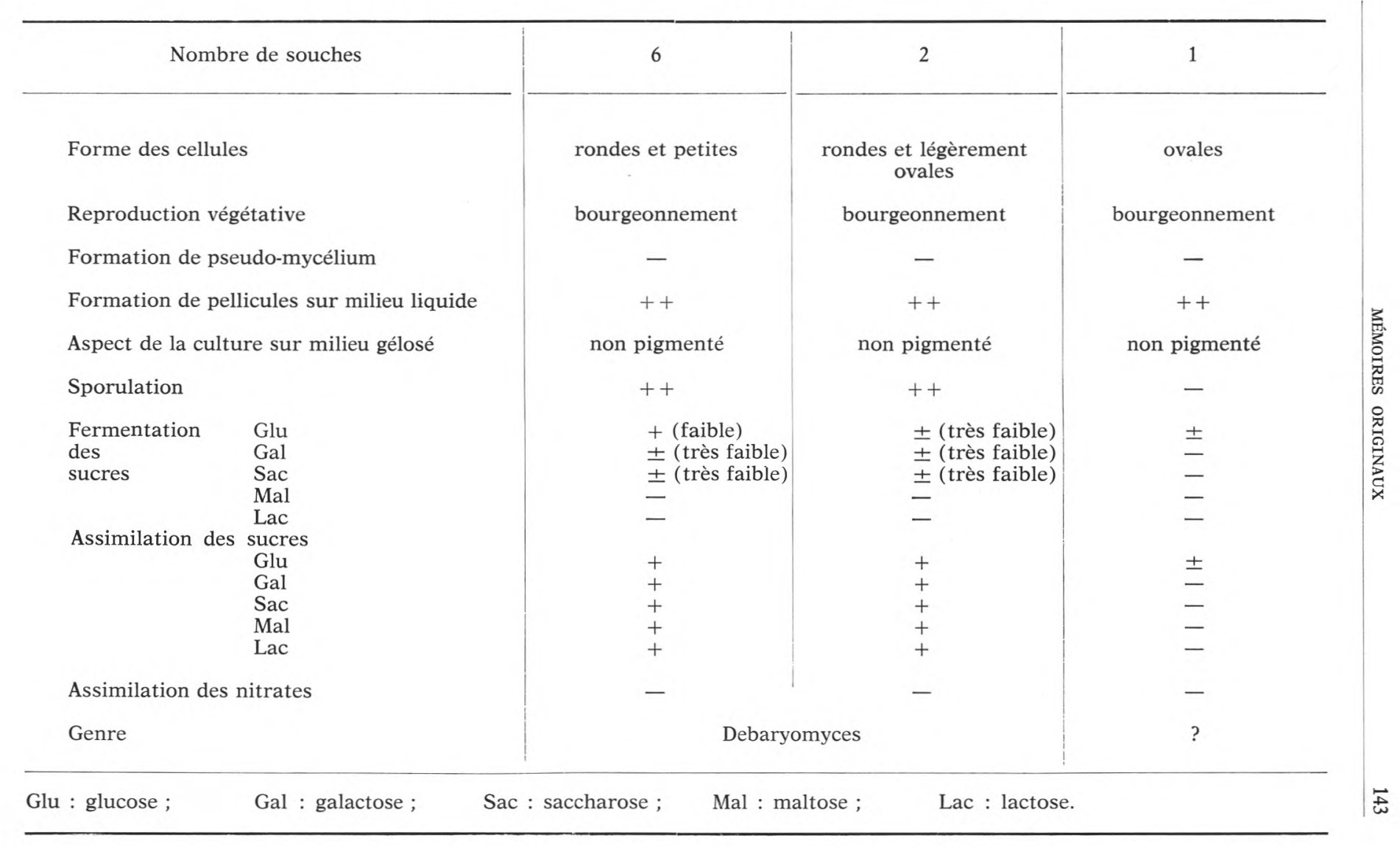




\section{IV. - DISCUSSION}

La flore microbienne est constituée essentiellement, au début de la fabrication, par des streptocoques lactiques ajoutés au lait pasteurisé avant l'emprésurage. Cela explique le nombre élevé de bactéries lactiques dénombrées sur le milieu spécial de Elliker et al. (1956), au début de la conservation, par rapport à la flore totale, car ce milieu n’est pas très spécifique.

$\mathrm{Au}$ cours de l'affinage du fromage, nous constatons que la flore lactique subit une destruction rapide et presque totale. Cette évolution est différente de celle que l'on observe dans d'autres fromages. Devoyod et al. (1968) avec le fromage de Roquefort, ont trouvé que les streptocoques lactiques forment toujours la flore dominante tout au long de l'affinage. Il est certain que la forte teneur en sel dans le fromage conservé en saumure et le taux d'acidité lactique élevé dans le fromage conservé sous «Cryovac» sont défavorables à la flore lactique.

En ce qui concerne l'évolution de la flore totale de la saumure, nous constatons que celle-ci est liée à celle des levures qui se trouvent en flore dominante à partir du $10^{\mathrm{e}} \mathrm{j}$ de conservation. On trouve que les levures représentent 93 p. 100 de la flore totale à la fin de l'affinage. Dans un fromage affiné en saumure les levures se développent également très vite.

L'étude que nous avons faite pour identifier les levures halophiles, survivant dans la saumure, nous a montré que celles-ci appartiennent au genre Debaryomyces. Des levures de ce genre ont été retrouvées par Ghonien (1968) dans le fromage "Domiati » qui est un bon exemple de fromage blanc salé et affiné en saumure. Devoyod et al. (1970) ont également isolé ces levures à partir du fromage de Roquefort. D'après ces auteurs, la forte teneur en chlorure de sodium (15 à $20 \mathrm{~g} \mathrm{de} \mathrm{NaCl}$ p. 100 de la phase aqueuse de la surface du fromage) a une action favorable sur le développement des levures appartenant aux genres Hansenula, Pichia et Debaryomyces.

Des inconvénients peuvent résulter de la présence de certaines levures. D'après Kiss et al. (1966) le bombement de boîtes de fromages blancs au cours du stockage en saumure est provoqué par des levures fermentant le lactose en éthanol et gaz carbonique. Ce n'est pas le cas des espèces du genre Debaryomyces que nous avons isolées car elles sont incapables de fermenter le lactose.

\section{Remerciements}

Nous remercions $M$. le Professeur Payens de l'ENSAIA de l'appui qu'il nous a donné pour l'identification des levures. 


\section{Rés u m é}

La flore totale du fromage conservé en saumure est constituée essentiellement, au début de la conservation, de streptocoques lactiques. La flore lactique subit ensuite une destruction rapide et presque entière. Les levures se trouvent à la fin de la conservation en flore dominante. Les bactéries coliformes ne se développent pas dans ces conditions.

Neuf souches des levures halophiles ont été isolées à partir de la saumure à 18 p. 100 de sel. Huit souches ont été identifiées comme appartenant à l'espèce Debaryomyces hansenii. Ces levures ne fermentent pas le lactose ; elles ne peuvent altérer le fromage et elles n'ont pas de signification hygiénique.

\section{S u m m a r y}

The microbiological aspect of pickled white cheese and of the brine was envisaged. The evolution of the microbical flora, the lactic streptococci and the yeast was investigated. The coliform organims were absent in all the products. At the end of curing the yeasts are predominant. Nine strains of yeasts were isolated from brine ; eight strains are belonging to the genus Debaryomyces.

\section{Bibliographie}

Аво EL-NAGA (I. G.) (1971). - Uber die Frühblähung von weissen Salzlakenkäse. Milchwissenchaft, 26, 747.

Elliker (P. A.), Anderson (A. M.) and Hannesson (G.) (1956). - Agar medium for lactic acid bacteria. J. Dairy Sci., 39, 1611.

GHoNIEM (N.A.) (1968). - Incidence of yeasts other than candida species in Domiati cheese. Milchwissenschaft, 23, 482.

Devoyod (J. J.), BRet (G.) et Auclair (J.E.) (1968). - La flore microbienne du fromage de Roquefort. Le Lait, 48, 613.

Devoyod (J. J.) et SPONEM (D.) (1970). - La flore microbienne du fromage de Roquefort. VI. Les levures, Le Lait, 50, 524.

Kiss (E.), Eross (E.) und Markus (P.) (1966). - Uber Bombage der Weisskäse enhaltenden Dosen. $X V I I^{\mathrm{e}}$ Congr. Int. Laiterie, $D, 1,143$.

LodDer (J.) and Kreeger van RiJ (N. J. W.) (1952). - The Yeasts. A taxonomic study. North Holland publishing Company, Amsterdam.

DE Man (J. C.), Rogosa (M.) and Sharpe (M. E.) (1960). - A medium for the cultivation of Lactobacilli. J. Appl. Bact., 23, 130.

Mansour (A.) (1972). - Etude biochimique et microbiologique du fromage affiné en saumure. Thèse (sciences), Nancy.

Mansour (A.) et Alais (C.) (1972 b). - Etude du salage et de l'affinage du fromage en saumure. Aspect biochimique : Evolution de la composition du fromage et rendement. Le Lait, 52, 515.

Mansour (A.) et Alais (C.) (1972, a). - Etude du salage et de l'affinage du fromage en saumure. II. Evolution chimique de la saumure. Le Lait, 52, 642.

Serres (L.), Amariglio (S.) et Petransxienne (D.) (1970). - Contrôle de la qualité des produits laitiers, T.2. Ministère de l'Agriculture, Services Vétérinaires, Paris. 